\section{Diffraction of Slow Positive Ions}

AN experimental method has been developed which permits photographic recording of the scattering of slow positive ions through angles of a fraction of a degree. This is accomplished by using a very fine, electrostatically focused incident beam and accelerating the ions, after the scattering, to a velocity high enough to affect a photographic plate. The highvoltage electrostatic lens used to give the acceleration also produces an angular magnification of the scattered rays.

Fig. 1 shows the scattering of lithium ions by a jet of sodium vapour, photograph $a$ for 292 volt ions, $b$ for 149 volt ions. Around the central spot is a sharply defined disk of nearly uniform intensity, having an angular radius of 0.0083 radians for the faster ions and 0.0151 for the slower ions. The plates also show a faint halo surrounding the disk and separated from it by about a quarter of the disk's diameter.

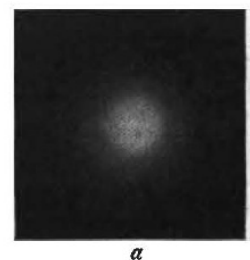

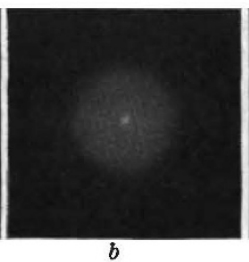

Fig. 1.

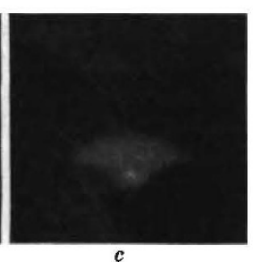

$c$
The pattern appears, at first sight, to resemble the diffraction of light by an opaque circular obstacle. However, a wave theory in which the atom and ion are treated as hard spheres gives a value of only $1 \mathrm{~A}$. for the collision radius and also predicts a wrong variation of disk radius with ion energy. The repulsive field model considered theoretically by Massey and Smith ${ }^{1}$ gives a monotonic decrease of elastic scattering with increasing angle and therefore also fails to account for the effects.

A satisfactory explanation of the results has been found which seems definitely to establish the wave nature of lithium ions. The essential features of the theory are the inclusion of the attractive force due to polarization of the sodium atom, and the division of the ion wave-front into three distinct parts corresponding, respectively, to exceedingly small, large and intermediate scattering angles.

The first part is taken in such a way that the retardation produced by the potential field of the atom is everywhere less than half a wave-length. Under these conditions there is negligible refraction of the wave-front and only slight diffraction corresponding to a large circular obstacle (in atomic units). This gives a central Airy's disk of about $0 \cdot 0006$ in angular radius, which is considerably less than the angular radius of the incident beam. The second or innermost part of the wave-front is where the retardation varies very rapidly, corresponding to large angle scattering.

The remaining annular part of the wave-front is what produces the observed pattern. Now, the calculations show that the retardation varies almost linearly across the width of this region and a hollow cone of rays would therefore be produced, were it not for diffraction. This has the effect of filling up the inside of the cone and also producing fringes, spaced in accordance with the width of the annulus.
The fringes inside the cone are smoothed out by overlapping, thereby giving the observed uniform disk. The separation of the halo from the edge of the disk is related directly to the width of the annulus.

The calculations have been made for a force of the form ${ }^{2}$

$$
F(r)=\frac{2 \alpha e^{2}}{r^{5}}\left[1-\left(\frac{r_{0}}{r}\right)^{4}\right]
$$

where $\alpha$ is the polarizability of the sodium atom and $r_{0}$ the equilibrium nuclear separation of $\mathrm{Na} \mathrm{Li} \div$. For both 292- and 149-volt ions the observed radii of disk and halo agree, within the limits of the experiment, with those calculated for $\alpha=2.5 \times$ $10^{-23} \mathrm{~cm} .{ }^{3}$ and $r_{0}=2 \cdot 36 \mathrm{~A}$. Since only two observations are needed to fix both $\alpha$ and $r_{0}$, this means that the theory correctly accounts for the observed variation of both radii with energy. The value of $\alpha$ fits in with the values found experimentally for $\mathrm{Li}, \mathrm{K}$ and $\mathrm{Cs}$ by Scheffers and $\mathrm{Stark}^{3}$, and $r_{0}$ is consistent with the spectroscopic values ${ }^{4}$ of $2 \cdot 67$ and 3.07 for the neutral molecules $\mathrm{Li}_{2}$ and $\mathrm{Na}_{2}$. From the values of $\alpha$ and $r_{0}$ the binding energy of $\mathrm{Na}$ $\mathrm{Li}+$ works out at 2.9 volts.

Fig. 1, $c$, shows the grazing-angle scattering of 292-volt lithium ions by a red-hot nickel target. As with sodium, there is a sharp intensity discontinuity, but the pattern has an approximately elliptical, and not circular, outer boundary.

A qualitative explanation of the effect is possible in terms of diffraction by single protruding surface atoms, using the same annular slit model as before, but with some allowance for the underlying atoms. The latter have the effect of smoothing out the potential behind the diffracting atom and consequently lessening the retardation gradient across the slit at that point. The pattern should accordingly be contracted in a direction normal to the target surface, as it actually is. It must be noticed that the retardation gradient across the slit bends the wavefront towards the atom, and therefore the part of the wave-front passing in front of the atom does not contribute to the pattern.

\section{A. G. Ensite.}

Laboratory of Physical Chemistry, Cambridge.

${ }^{1}$ Proc. Roy. Soc., A, 142, 142 (1933).

${ }^{2}$ Hassé and Cook, Phil. Mag., 12, 554 (1931).

${ }^{3}$ Phys. Z., 35, 625 (1934).

"Sponer, "Molekülspektren, 1", Tabellen.

\section{Vibration Temperature in relation to Rotation Temperature in Band Spectra}

THE problem of exciting band spectra of two different molecules in the same source has attracted some attention of late. Investigation of excitation temperatures of molecules under these conditions has given interesting results which require elucidation from theory. For example, Lochte-Holtgreven ${ }^{1}$ found by examining $\mathrm{CH}$ and $\mathrm{C}_{2}$ bands in the same discharge tube a temperature difference of more than $1,000^{\circ}$ between the two. On the other hand, van Wijk ${ }^{2}$ arrived at a common effective temperature from the negative $\left(\mathrm{N}_{2}^{+}\right)$and second positive $\left(\mathrm{N}_{2}\right)$ bands of nitrogen in the same discharge tube. In view of these results, it was deemed necessary to undertake further work on the same lines.

The present investigation deals with AlO bluegreen and $\mathrm{CN}$ violet systems excited in the same 